\title{
A multiscale DEM-LBM analysis on permeability evolutions inside a dilatant shear band
}

\author{
WaiChing Sun $\cdot$ Matthew R. Kuhn • \\ John W. Rudnicki
}

Received: 26 October 2012/Accepted: 16 January 2013/Published online: 31 May 2013

(C) Springer-Verlag Berlin Heidelberg (outside the USA) 2013

\begin{abstract}
This paper presents a multiscale analysis of a dilatant shear band using a three-dimensional discrete element method and a lattice Boltzmann/finite element hybrid scheme. In particular, three-dimensional simple shear tests are conducted via the discrete element method. A spatial homogenization is performed to recover the macroscopic stress from the micro-mechanical force chains. The pore geometries of the shear band and host matrix are quantitatively evaluated through morphology analyses and lattice Boltzmann/finite element flow simulations. Results from the discrete element simulations imply that grain sliding and rotation occur predominately with the shear band. These granular motions lead to dilation of pore space inside the shear band and increases in local permeability. While considerable anisotropy in the contact fabric is observed with the shear band, anisotropy of the permeability is, at most, modest in the assemblies composed of spherical grains.
\end{abstract}

\footnotetext{
W. Sun $(\bowtie)$

Mechanics of Materials, Sandia National Laboratories, Mail Stop 9912, 7011 East Avenue, Livermore, CA, USA

e-mail:wsun@sandia.gov

M. R. Kuhn

Department of Civil Engineering, Donald P. Shiley School of Engineering, University of Portland, 500 N. Willamette Blvd, Portland, OR 97203, USA

J. W. Rudnicki

Department of Civil and Environmental Engineering,

Technology Institute A333, Northwestern University,

2145 Sheridan Road, Evanston, IL 60208, USA
}

Keywords Discrete element method · Homogenization · Lattice Boltzmann method - Micromechanics of granular materials $\cdot$ Microstructure $\cdot$ Strain localization

\section{Introduction}

Deformation bands in geological materials are narrow zones of inhomogeneous strain. Their development is often analyzed as a material bifurcation phenomenon that takes place as a deviation from homogeneous deformation [5, 34]. Deformation bands can be categorized as shear, compaction, or dilation, depending on the predominant mode of localized strain within the band. In geological materials, shear occurs most commonly with some amount of volumetric deformation, either dilation or compaction $[3,7,11,33,40]$. For instance, compactive shear bands, thin zones with reduced porosity and dominated shear, have been reproduced in sand experimentally to study the role of spatial variability of relative density on the onset, propagation, and orientation of shear bands in [7]. On the contrary, a dilatant shear band can cause an increase in porosity and change of flow path, thereby altering the fluid transport inside the pore space. This feature is of economic importance, because dilatant shear bands may act as flow conduits that leak injected pore-fluid in reservoirs [44].

To predict how formation of dilatant shear bands affects the hydraulic properties, macroscopic finite element analysis may be used to capture the evolution of porosity. Changes of permeability are then estimated from the porosity evolution via empirical or semi-empirical constitutive relations. This macroscopic approach has several drawbacks. First of all, empirical models, such as capillary tube model [36] and fissure model [19], are often designed and validated for very specific types of materials with 
relatively simple pore geometry. Second, empirical models, such as the modified Kozeny-Carman equation [4], are often designed mainly to capture the macroscopic porosity-permeability relation, while permeability changes due to macroscopic shear and grain rearrangement, rotation, and sliding are neglected. Hence, a macroscopic empirical porosity-permeability relation may fail to deliver reliable predictions when the permeability changes are caused by factors other than volumetric changes of pore space, such as isolation of the pores and changes in tortuosity and size distribution of flow channels, as shown in [39].

Volumetric digital image correlation (VDIC) applied to $\mathrm{X}$-ray tomography images of geomaterials may help in understanding the kinematics of grain motion and changes in pore geometry as shown in $[27,32]$. However, due to the technical difficulty of conducting mechanical tests while simultaneously performing CT X-ray imaging, experimental measurement of microstructural attributes is rare (e.g., $[1,16])$. Furthermore, while continuum kinematics quantities such as strain can be extracted via VDIC [7, 11], tracking motion and deformation of individual grains is still a computationally demanding process [1]. Recently, Andò et al. [1] propose a discrete particle ID approach to directly identify and keep track of individual grains without the necessity of image analysis and hence significantly improve the speed. However, post-processing procedures are required to correct the error due to incorrect tracking of grains with similar geometrical features.

DEM is widely used to model particle assemblies and to simulate the mechanical behavior of granular materials. Recent work has coupled DEM with LBM and finite element models to study fluid flow processes that evolve on a similar time scale as particles are being rearranged: soil liquefaction, fluidized beds, and suffusion $[8,14,15]$. In this study, our focus is on shear band formation in highporosity granular material under the fully drained condition. Because the shearing that produces shear bands in sands is slow in comparison with fluid migration under the drained condition, the current work decouples the DEM and LBM analyses, with DEM used to simulate the formation of a band and LBM analysis used to analyze the fluid flow after formation of the band.

To analyze the links between microstructural granular motion and macroscopic hydraulic properties in a repeatable and cost-efficient way, we introduce a multiscale numerical alternative. This approach incorporates the discrete element method (DEM) to simulate grain motion inside the dilatant shear band and a multiscale lattice Boltzmann (LBM)/finite element (FEM) scheme to capture the evolution of permeability. Since the motion of every particle in the DEM ensemble is recorded at every time step, this approach makes it possible to quantify how individual particles affect macroscopic hydraulic properties without the necessity of introducing an additional phenomenological law.

As for notations and symbols, bold-faced letters denote tensors; the symbol ' $'$ ' denotes a single contraction of adjacent indices of two tensors (e.g., $a \cdot b=a_{i} b_{i}$ or $c \cdot d=c_{i j} d_{j k}$ ); the symbol ' $:$ ' denotes a double contraction of adjacent indices of tensor of rank two or higher (e.g., $\left.C: \varepsilon^{e}=C_{i j k l} \varepsilon_{k l}^{e}\right)$; the symbol ' $\otimes$ ' denotes a juxtaposition of two vectors (e.g. $a \otimes b=a_{i} b_{j}$ or two symmetric secondorder tensors (e.g., $(\alpha \otimes \beta)=\alpha_{i j} \beta_{k l}$ ).

\section{Method}

\subsection{Grain assembly from simple shear discrete element simulation}

In this study, we use the open source 3D discrete element (DEM) code OVAL to simulate a simple shear test on a specimen composed of spherical particles [2, 13, 25]. A DEM simulation represents a granular material as an assembly of elemental particles, among which interactions are explicitly modeled by simplified constitutive contact laws [13]. Each particle's motions are obtained through force and momentum balances, expressed as

$m \ddot{u}+C^{m} \dot{u}+P(u)=F_{\mathrm{ext}}$

$I \cdot \ddot{\omega}=M_{\mathrm{ext}}$

where $m$ and $C^{m}$ are the mass and translational damping. Vector $P(u)$ is the net force imbalance vector due to the inter-particle contact forces, and $F_{\text {ext }}$ denotes the external loads exerted by walls or gravity. $I$ is the moment of inertia, $\ddot{\omega}$ is the rotational acceleration, and $M_{\text {ext }}$ is the external moment on a particular grain. For simplicity, gravity loads are neglected in the current discrete element simulations. To solve Eq. 1, a time-stepping explicit central difference leap-frog algorithm is used [13]. When simulations are intended to approximate slow, quasi-static loading conditions, the mass damping $C^{m}$ and contact damping $C^{s}$ (described below) must be sufficient to dissipate high frequency vibrational modes without unduly impeding particle motions that arise from particle interactions or the boundary conditions. The homogenized macroscopic stress and strain tensors, $\boldsymbol{\sigma}$ and $\boldsymbol{\varepsilon}$, of the granular assembly are determined from the contact forces $f_{i}^{\alpha}$ and branch vectors $l_{j}^{\alpha}$ between the centers of the $N_{c}$ contacting particle pairs and from the average deformation gradient $F_{i j}$ of the assembly:

$\sigma_{i j}=\frac{1}{V_{\Omega}} \sum_{\alpha=1}^{N_{c}} f_{i}^{\alpha} l_{j}^{\alpha} ; \quad \varepsilon_{i j}=\frac{1}{2}\left(F_{i k} F_{k j}-I_{i j}\right)$

where $V_{\Omega}$ is the assembly volume. 


\subsubsection{Contact model}

In our implementation, we use a simplified contact model in which the grain-to-grain force-displacement relation is governed by a frictional Hertz-Mindlin mechanism with viscous damping but without cohesive bonding between particles. Incremental changes in the normal and tangential contact forces, $f^{\mathrm{n}}$ and $f^{\mathrm{t}}$, are determined by the shear modulus of the grains $G_{g}$, Poisson ratio $v$, radii of the contacting grains $R_{1}$ and $R_{2}$, and the normal and tangential displacements at the contact, $\mathrm{d} \delta$ and $\mathrm{d} s$ [21],

$d f^{n}=k^{n} \mathrm{~d} \delta ; \quad k^{n}=\frac{\sqrt{2} G_{g} \sqrt{R^{e}}}{1-v} \delta^{1 / 2}$

$d f^{t}=k^{s} \mathrm{~d} s ; \quad k^{s}=\frac{2 \sqrt{2} G_{g} \sqrt{R^{e}}}{2-v} \delta^{1 / 2}$

where $\delta$ is the indentation at the contact and $R^{e}$ is the effective radius,

$R^{e}=\frac{2 R_{1} R_{2}}{R_{1}+R_{2}}$

The tangential force is limited by the friction coefficient $\mu^{f}$, such that $\left|f^{t}\right| \leq \mu^{f} f^{n}$. Equation 5 gives the initial, elastic tangential stiffness $k^{s}$ between elastic spheres. The incremental tangential force $f^{t}$, however, is path-dependent, and an exact solution involves the history of normal and tangential force at the contact [24]. The approximate solution of Thornton and Randall [43] is used in the current simulation, which permits solution of a full loading-unloading-reloading cycle between two spherical grains.

To maintain a stable quasi-static simulation, a viscous damping force is applied at each contact

$f^{s, \text { vis }}=C^{s} \dot{s}$

where $\dot{s}$ is the tangential sliding velocity at a contact, and $C^{s}$ is the contact viscosity. This contact viscosity is "turned off" whenever frictional sliding occurs, as such sliding becomes a dissipation mechanism that prevents spurious grain vibrations. Mass (global) damping $\left(C^{m}\right.$ in Eq. 1) can delay or prevent the emergence of shear bands, since an emerging difference in velocities on either side of the band imparts a reactive viscous force that will suppress the difference. To freely permit the spontaneous emergence of shear bands, the viscosity $C^{m}$ was set to zero, and the contact (local) viscosity $C^{s}=0.12 \sqrt{m k^{n}}$ was used exclusively as a damping measure. This small viscosity was sufficient to maintain a stable simulation without unduly affecting shear band thickness, since thickness is insensitive to the contact damping parameter, just as it is insensitive to the contact friction coefficient [23]. In this manner, grain size and shape were the dominant factors controlling the emergence and character of the shear band. In the simulation, the shear modulus $G$ was $29 \mathrm{GPa}$, the Poisson ratio $v$ was 0.15 , and the friction coefficient between particles was 0.50 . These values are in the ranges of those measured for quartz grains [30].

\subsubsection{Initial particle arrangement}

The DEM simulation was conducted with an assembly of 129,000 spherical grains that were densely arranged with an initial isotropic fabric. The distribution of particle sizes is shown in Fig. 1 and corresponds to a poorly graded medium sand with a coefficient of uniformity $C_{u}$ of 1.4 ( $=D_{60} / D_{10}$ ), a median particle size $D_{50}$ (by volume) of 1.00 $\mathrm{mm}$, and an effective size $D_{10}$ of $0.754 \mathrm{~mm}$. The dense arrangement was attained from an initially sparse random arrangement of particles by isotropically reducing the boundary dimensions. During compaction, friction was removed from the particle interactions, a technique that is commonly used to achieve high packing densities [42]. The compacted porosity was 0.337 (a void ratio of 0.509 ), with an initial average coordination number of 5.52 contacts per particle. Because of the isotropic compaction technique, the initial normal stresses in all directions were nearly equal to the mean stress of $416 \mathrm{kPa}$. These stresses, computed with Eq. 3, are the inter-granular, effective stresses that are developed by the contact forces.

\subsubsection{Boundary conditions}

The assembly shape and boundary conditions were chosen to promote the free emergence of shear bands during simple shear loading, and the assembly dimensions were chosen to produce a shear band of sufficient volume for studying its fabric and hydraulic properties. The DEM assembly dimensions were $50.6 \times 118 \times 12.7 \mathrm{~mm}$, or in terms of the particle size $D_{50}$, about $51 \times 118 \times 13 D_{50}$. Shearing was imposed across the $118 \mathrm{~mm} y$-height by

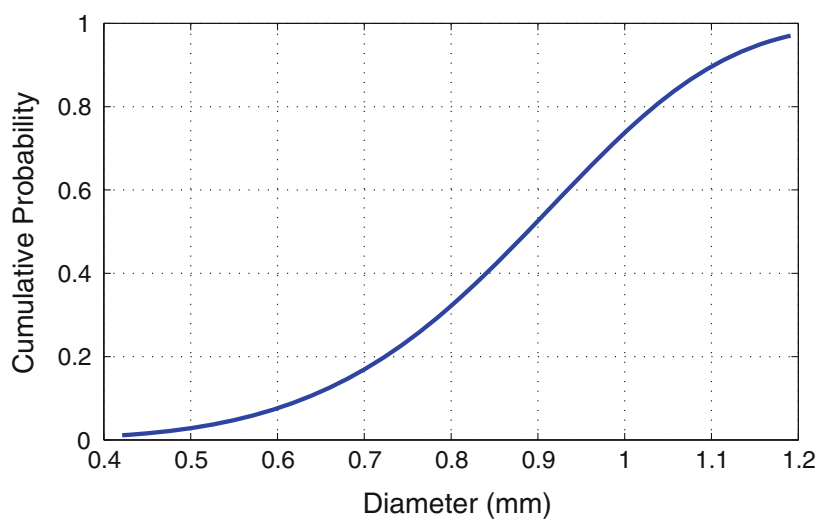

Fig. 1 The cumulative probability function of grain diameter of the DEM assembly 


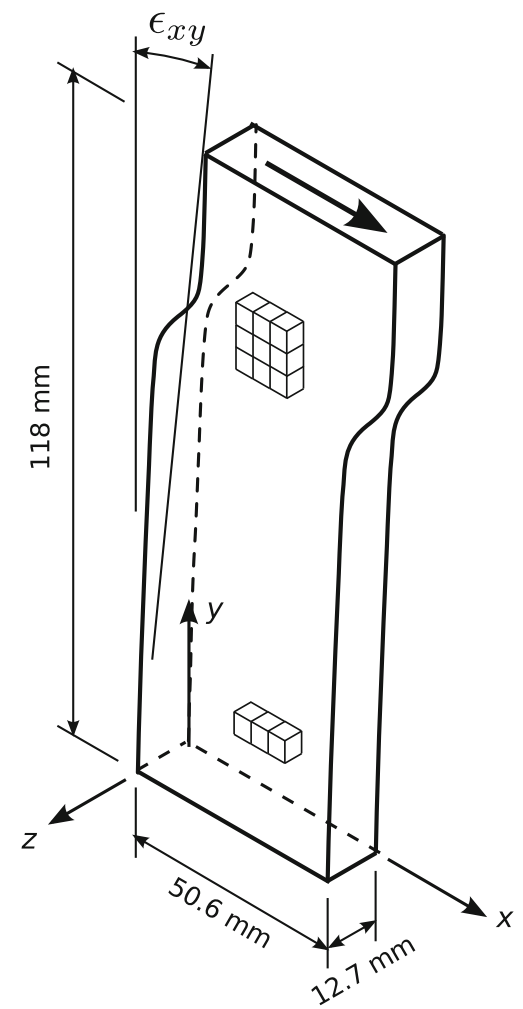

Fig. 2 DEM assembly and loading conditions. Small boxes show locations of the $4 \mathrm{~mm}$ unit cells used for pore-fluid analysis (Sect. 3.2)

moving the top boundary horizontally at a constant velocity in the $x$-direction (Fig. 2), while maintaining constant assembly widths in the $x-x$ and $z-z$ directions and a constant vertical stress $\sigma_{y y}$. That is, the vertical height was freely allowed to change while the assembly was undergoing simple shear at a constant shearing rate and under constant vertical stress. Periodic boundaries were used on all sides of the assembly during the initial compaction process [12], but the periodicity of the top and bottom boundaries was broken at the start of shearing and replaced with thin rigid horizontal layers of conforming spheres that were arranged along the $x-z$ planar directions. In this manner, the top and bottom boundaries were modeled as rough rigid platens. The four vertical sides, in $x-y$ and $y-$ $z$ planar directions, remained as periodic boundaries during shear loading. With these conditions, a shear band could develop along the full $x-x$ width and $z-z$ depth, while passing across the periodic side boundaries.

\subsection{Geometrical analysis on pore geometry of simulated assembly}

To analyze how grain rearrangement affects the hydraulic property of the assembly, we convert portions of the 3D DEM assembly into 3D discrete binary images. The binary images are collections of binary voxels which signal whether a cubic volume is occupied by solid $(b=255)$ or by void $(b=0)$. The union of void voxels can be used as the simulation domain for lattice Boltzmann flow problems. In addition, binary images can be analyzed with software designed for tomographic images, such as ImageJ [37].

We use a simple computer algorithm called seed-fill to create the binary images of the DEM assemblies. As the name implies, the seed-fill algorithm is used to grow the region from a seed such that the "flag" or "identification" of the seed can be propagated in a confined object. The seeds we used in the problem are the centroids of the spherical particles. To obtain the binary image of the DEM assemblies, we confine the growing of the seeds by preventing them from growing outside the spherical particles. The pseudo-code used to create binary image goes as follows, noting that the algorithm is recursive. A similar algorithm has been used in [40] to determine the connected/isolated porosity of Aztec sandstone. To quantitatively compare the geometrical features of the pore space inside and outside the shear band, we extract a set of geometrical parameters, i.e. the Euler number and the

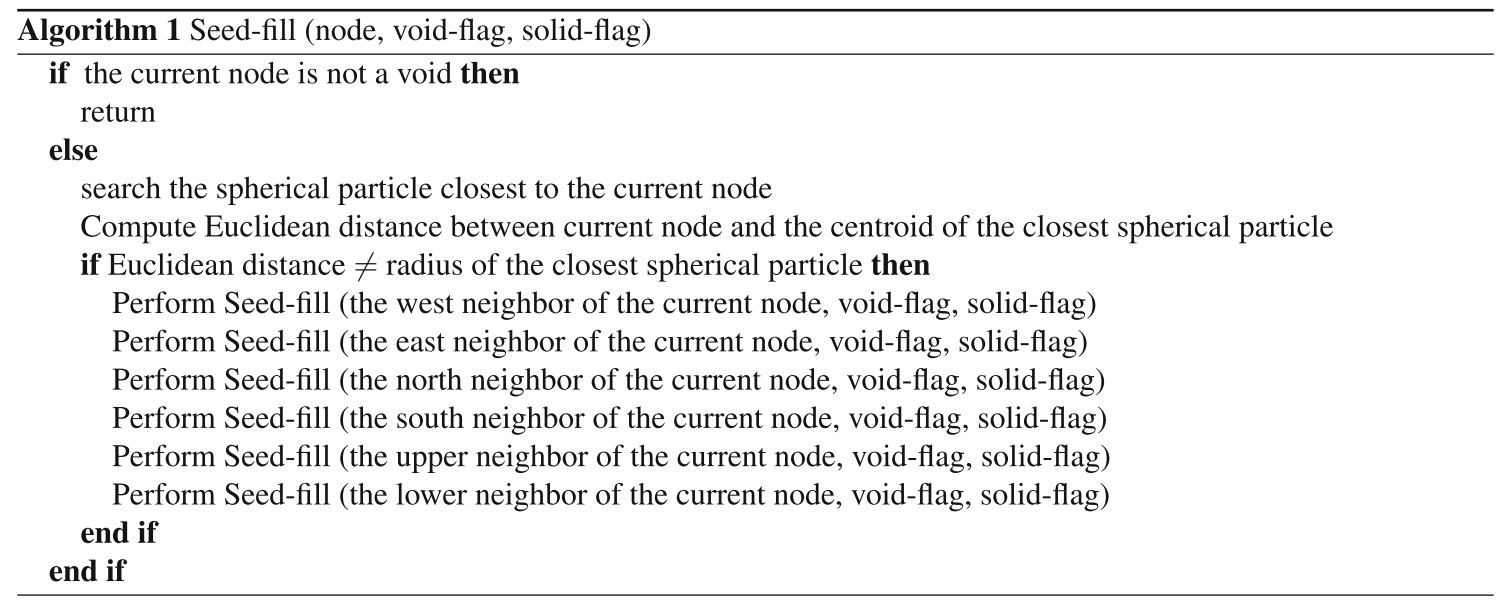


surface area/pore volume, which are found to be closely related to hydraulic transport behavior of porous media [17, $22,28,37]$. The open source computer algorithm used to extract these parameters is provided by Legland [26]. The Euler number of binary image $\mathcal{E}$ is defined as,

$\mathcal{E}=N-C+H$

where $N$ is the number of interconnected pores, $C$ is the number of loops in the pore space, and $H$ is the number of objects completely enclosed by pore space. In other words, the Euler number is a measure of connectivity which is positive if the pores are poorly connected and negative if otherwise. A large negative Euler number indicates that the pore space has a network-like topology and that there are more possible flow paths for pore-fluid traveling from one end to another end. In addition, tortuosity of flow channels can be measured by computing the ratio between the surface area and the pore volume. Obviously, a high surface area/pore volume indicates that pore-fluid must travel along a longer flow path from one end to the other.

\subsection{Geometrical enhanced lattice Boltzmann/finite element simulation}

A multiscale lattice Boltzmann/Finite element method is used to extract geometrical features and permeability from the granular assembly. This hybrid method was originally proposed in [45] to estimate permeability of Castlegate Sandstone. Sun et al. [39] improved the accuracy and computation efficiency of this method by incorporating geometrical analysis in the permeability calculations. The key to this improvement is partitioning the entire grain assembly into unit cells where pore-scale lattice Boltzmann simulations are conducted in the connected pores of each unit cell. Since discretized voxel images may contain isolated pores if the resolution is insufficient, this method is used to ensure that discretization does not alter connectivity of the pores.

The lattice Boltzmann method we used is a single-relaxation time Bhatnagar-Gross-Krook (BGK) model [10, 38], which solves a discretized Boltzmann equation by simulating the evolution of fluid particle distribution that propagates and collides locally among lattice nodes. The macroscopic fluid flow is then approximated by the overall particulate motion of the fictitious fluid particles. The evolution of fluid particle distribution function $f_{i}$ in direction $e_{i}$ is updated in each time step through the following equation,

$f_{i}\left(x+e_{i}, t+\Delta t\right)-f_{i}(x, t)=-\frac{1}{\tau}\left(f_{i}(x, t)-f_{i}^{e q}(x, t)\right)$

where $f^{\mathrm{eq}}$ is a truncated equilibrium distribution defined as

$f_{i}^{\mathrm{eq}}=w_{i} \rho\left(1+\frac{3 e_{i} \cdot v}{c_{s}^{2}}+\frac{9\left(e_{i} \cdot v\right)^{2}}{c_{s}^{4}}-\frac{v \cdot v}{2 c_{s}^{2}}\right)$ $\tau$ is a parameter related to the dynamic fluid viscosity $v$, as shown in the Chapman-Enskog equation (cf. [38]),

$v=\Delta t c_{s}^{2}\left(\tau-\frac{1}{2}\right)$

where $c_{s}$ is the constant speed of sound. $\rho$ and $v$ are the macroscopic density and velocity. They are determined from the evolution of the particle distribution, i.e.,

$\rho=\sum_{i=1}^{N} f_{i} ; \quad v=\frac{1}{\rho} \sum_{i}^{N} f_{i} e_{i}$

The effective permeability of a porous medium can be measured by applying a pore pressure gradient along a basis direction and determining the resultant fluid filtration velocity from a pore-scale hydrodynamics simulation. Then, the effective permeability tensor is obtained according to

$k_{i j}=-\frac{\mu^{v}}{\left(\nabla^{x} p\right)_{j}} \frac{1}{V_{\Omega}} \int_{\Omega} v_{i}(x) \mathrm{d} \Omega$

where $\mu^{v}$ is the kinematic viscosity of the fluid occupying the spatial domain of the porous medium $\Omega$. To implement this procedure, we first assume that the effective permeability tensor $k_{i j}$ is symmetric and positive definite. Assuming that the principal axes of the permeability tensor are aligned with the specimen axes, we determine the diagonal components of the effective permeability tensor $k_{i i}$ by three hydrodynamics simulations. In each simulation, we impose a pressure gradient on two opposite faces orthogonal to an axis $e_{i}$ and no-slip boundary conditions on the four sides parallel to $e_{i}$ where $e_{i}$ denotes an orthogonal axis of a Cartesian system. This numerical treatment has been previous used in [45] to estimate permeability of Castlegate sandstone.

Another possible approach to estimate permeability is by assigning uniform force in (9) at each lattice node such that a gravitational body force leads to fluid flow [38, 41]. This method may minimize the impact of artificial boundary effect. However, this approach only works for periodic media. Hence, non-periodic media must be made periodic by specular reflection on its boundary. This technique requires careful pre-programming to handle complicated local metrics, such as corners. As a result, this approach is not used in this study, but will be considered in future work.

\section{Results and discussion}

3.1 Microstructural attributes and homogenized constitutive responses

The objective of the numerical experiments is to explore how inter-particle motions, such as grain sliding and 
rotation and rearrangements of grains, influence macroscopic mechanical behavior and hydraulic properties of the shear band. In real geomaterials, there are numerous other microstructural attributes (e.g. grain size distribution, grain shapes, grain surface roughness, scale of fluctuations of mechanical properties) and mechanisms (e.g. grain sliding, rotation, crushing, interlocking) that may influence the overall macroscopic behavior in various degrees of importance. Since the influences of these factors are difficult to analyze simultaneously, we restrict our focus to the effects of grain sliding and rotation. To minimize the influence of inherent heterogeneity, all grains in the granular assembly are spherical with the distribution shown in Fig. 1.

\subsubsection{Stress-strain response}

Figure 3 shows the stress-strain response of the grain assembly during simple shear loading with constant vertical stress $p_{\mathrm{o}}=416 \mathrm{kPa}$. The peak shear stress occurs at shear strain $\varepsilon_{x y}=4.4 \%$. After reaching this peak, softening occurs as the shear stress decreases from 277 to around $170 \mathrm{kPa}$ at $\varepsilon_{x y}=9 \%$. At strains beyond $9 \%$, the shear stress fluctuates at $170 \mathrm{kPa}$, signifying a critical-state, residual condition that continues even beyond $12 \%$ strain, to strain of $20 \%$, the terminal strain in the simulations. The results correspond to peak and residual friction angles $\phi$ of $33.6^{\circ}$ and $22.2^{\circ}$, respectively. These strengths are somewhat smaller than would be expected with uncemented sands, a result of the smooth spherical particle shapes. A similar trend is observed in a plot of the mean stress $\left(\sigma_{x x}+\sigma_{y y} 0+\sigma_{z z}\right) / 3$ (see Fig. 4), with the mean stress reaching a maximum value at strain $\varepsilon_{x y}=3.8 \%$ and then remaining nearly level until it abruptly decreases between the strains $\varepsilon_{x y}=6 \%$ and $8 \%$. The average mean stress then fluctuates at $440 \mathrm{kPa}$ during the residual condition, about $6 \%$ larger than the initial confinement stress. These changes in the mean stress result from changes in

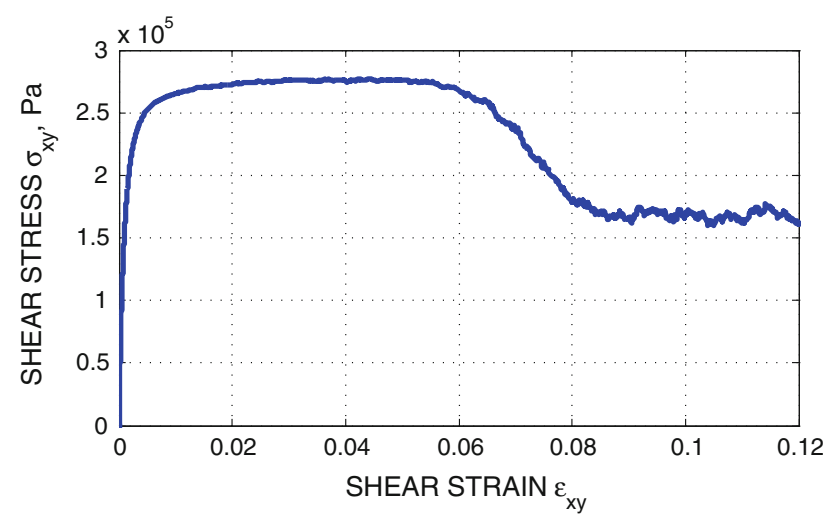

Fig. 3 Shear stress-strain response during simple shear loading horizontal confinement, $\left(\sigma_{x x}+\sigma_{z z}\right) / 2$, since the vertical stress $\sigma_{y y}$ is maintained a constant $416 \mathrm{kPa}$ throughout shearing.

Even as the compressive mean stress increases, the volume and porosity monotonically increase during shearing and then remain constant after the shear stress decreases to the residual level (Fig. 5). This trend is consistent with the plastic dilatancy commonly observed in dense spherical packings and in dense sands. Casagrande [9] attributed this increase in volume to the re-arrangement of densely packed particles as they rise up over neighboring particles. This phenomenon may be absent in loose packings, in which shear may induce pore collapse and densify the assembly.

Figure 6 shows the average coordination number of the entire assembly (the average number of contacting neighbors per particle) and the percentage of contacts that are sliding. The results are slightly skewed by the top and bottom layers of rigidly moving platen particles, which have only interior neighbors and do not slide among themselves. The average coordination number decreases greatly in the early, pre-peak strains as particles disengage

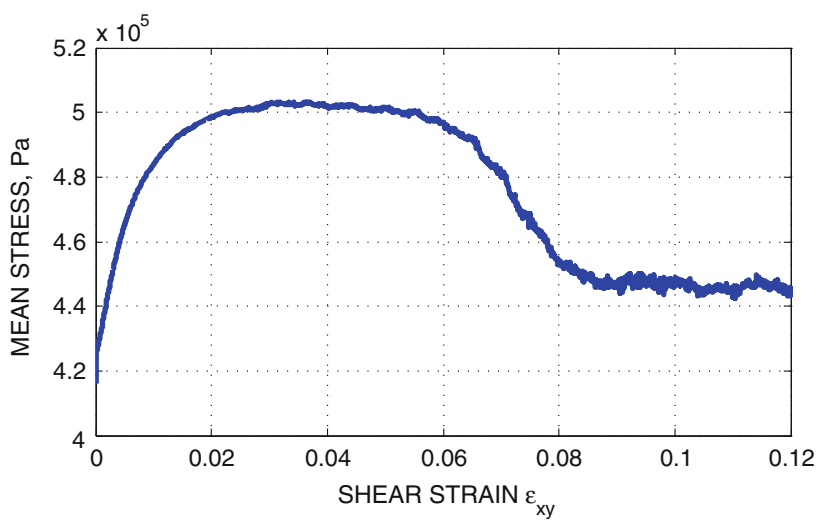

Fig. 4 Mean stress versus shear strain during simple shear loading. Compressive stress is positive

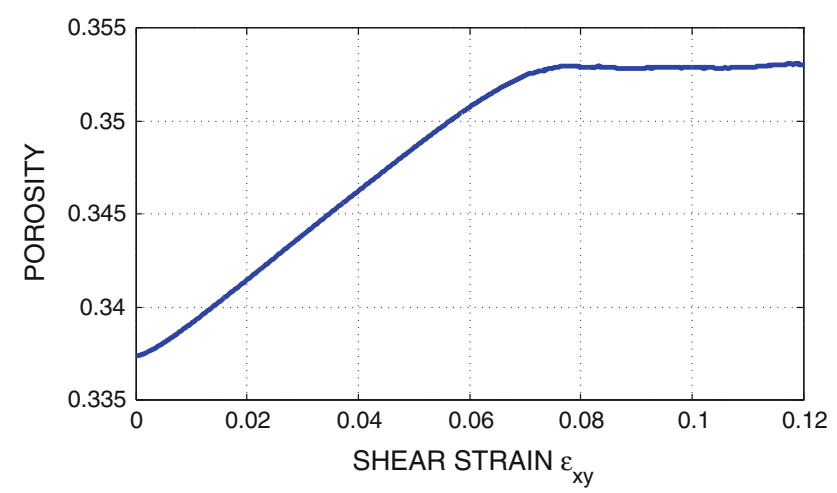

Fig. 5 Porosity versus shear strain during simple shear loading 
during this formative dilational stage. As shearing stress approaches the peak, the number of contacts decreases more slowly and remains nearly constant throughout the subsequent peak, softening and residual periods (a trend also observed by Thornton [42]). Figure 6 also shows that frictional sliding is mobilized early in the loading process, with the fraction of sliding contacts reaching a maximum of $29 \%$, well before the peak stress is attained. The fraction of sliding contacts then decreases to about $18 \%$ while the shearing stress is near its peak state. This decrease in the number of sliding contacts suggests that deformation begins to localize before the peak state is reached and before a shear band is fully developed. Once a shear band has formed and the material is softening (at strains greater than $5 \%$ ), the fraction of sliding contacts decreases again to less than $10 \%$ during the remainder of loading. In this final stage of loading, deformation and sliding are concentrated in the small, localized volume of the shear band.

\subsubsection{Localized deformation in shear band}

Localized deformation is apparent in Fig. 7, a view of the $x-y$ plane through the full $z$-depth of the assembly. The figure shows the overall deformed profiles and the distributions of individual particle rotations about their $z$-axes, $\omega_{z}$. At a pre-peak strain of $\varepsilon_{x y}=2 \%$, the deformed, tilting profile is nearly uniform, although rotations, which are both positive and negative, hint at intensely non-uniform motions at the micro-scale of individual particles. A shear

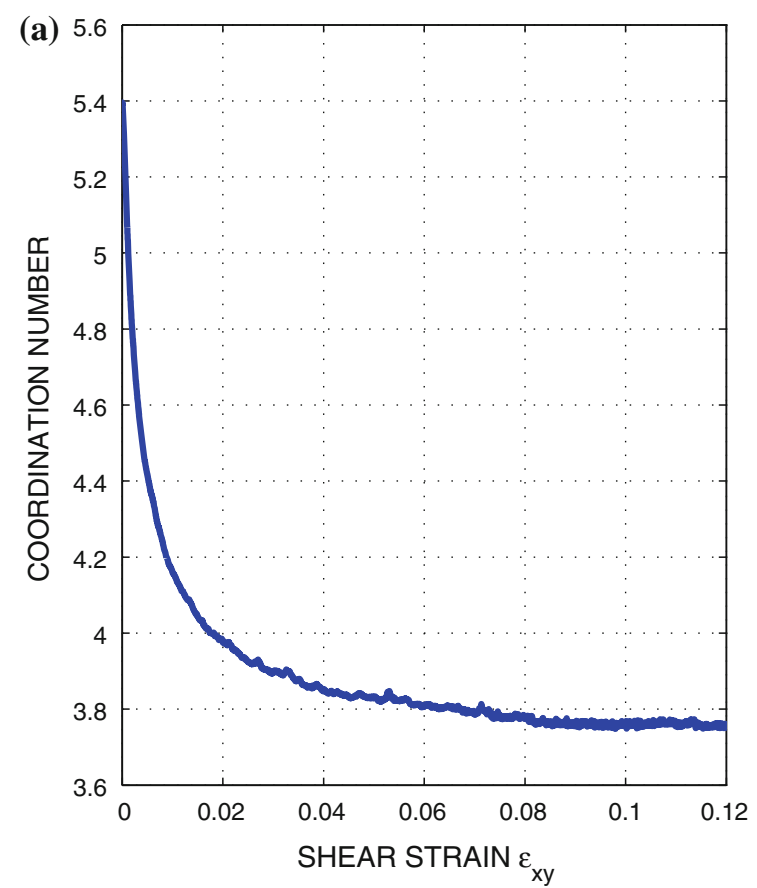

band is clearly forming within the upper half of the assembly at $6 \%$ strain. Less apparent are other non-persistent, ephemeral localization bands that occur between strains of 4 and $6 \%$, near the peak stress condition. At strains greater than $6 \%$, continued deformation coalesces within the upper shear band. Although grain rotation is more uniform prior to formation of the shear band, rotations become concentrated within the band, with some grains rotating about 19 radians (over three full rotations). The shear band, occurring along a horizontal plane, is located at about three-quarters of the assembly height from the base of the assembly. In simulations on similar assemblies, the band was found to occur at other heights, the particular location being unpredictable and likely the result of subtle weaknesses within the assembly.

The nature of stress and deformation within the band are illustrated in Fig. 8 which shows profiles of porosity, shearing strain and rotation, and confinement stress at the bulk strain $\varepsilon_{x y}=12 \%$. Together, the three plots indicate that the shear band is about $13-16 \mathrm{~mm}$ thick, or about 13-16 times $D_{50}$. Porosity is clearly larger within the shear band than elsewhere within the profile (Fig. 8a). Indeed, historical awareness of such localization was based upon $\mathrm{x}$-ray transmission images showing thin planar features of reduced density $[6,11,29]$. By comparing Figs. 3, 5, 7 and $8 \mathrm{a}$, we see that softening and dilation become concentrated within the shear band at strains of 5-8\%, after which, material within the shear band attains a steady, criticalstate density. Outside of the shear band, the density retains

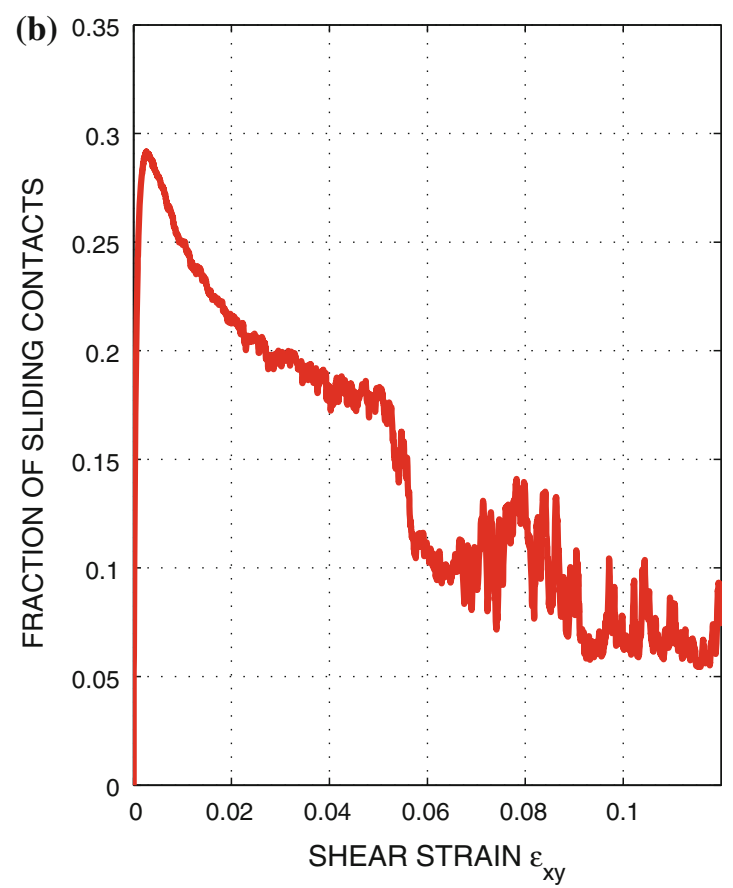

Fig. 6 a Evolution of coordination number and $\mathbf{b}$ fraction of sliding contacts during simple shear loading 


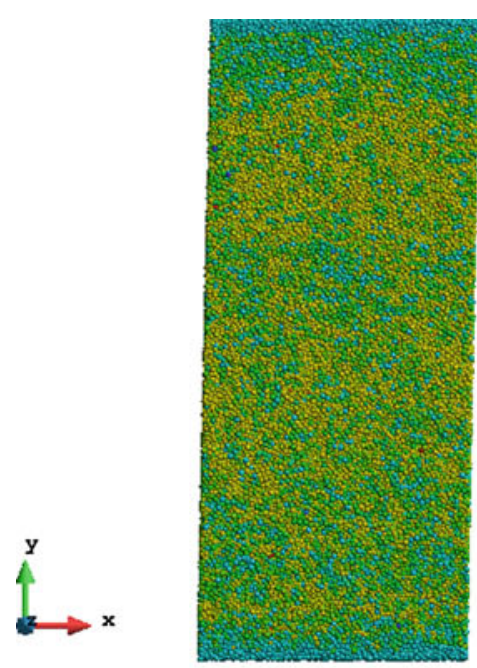

(a) 2\% shear strain

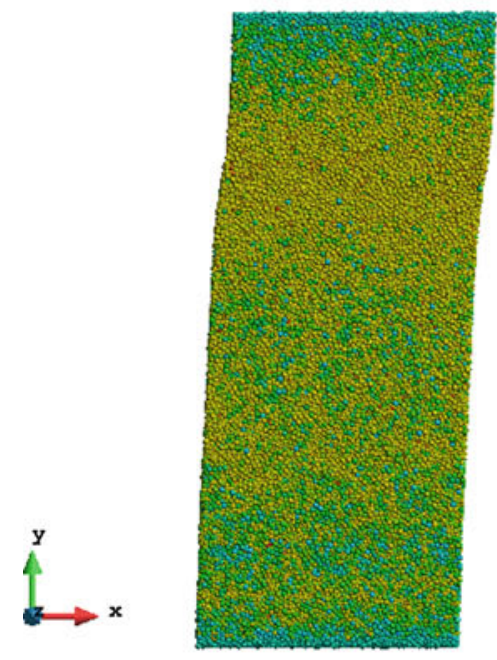

(c) $6 \%$ shear strain

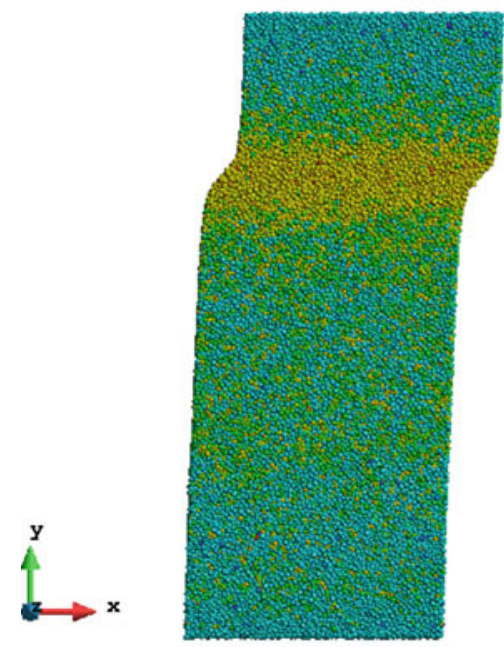

(e) $10 \%$ shear strain

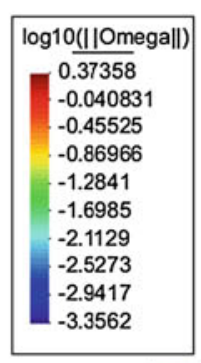

GiD

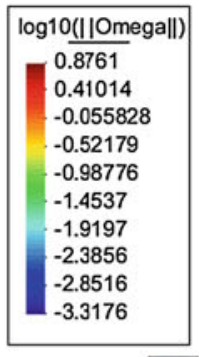

GiD
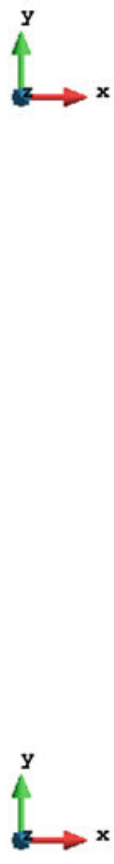$$
\text { . }
$$

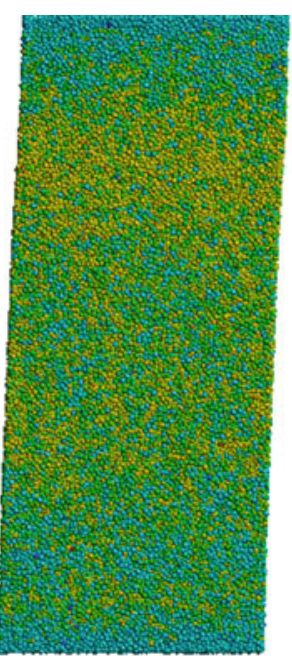

(b) $4 \%$ shear strain

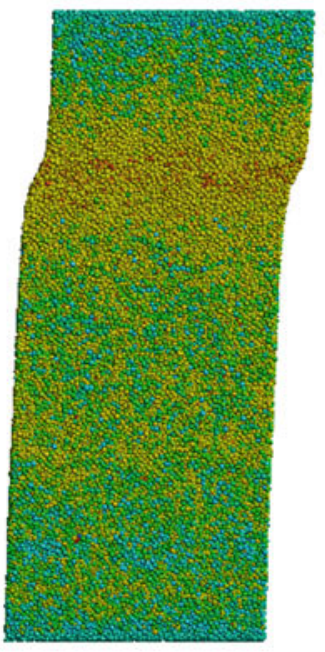

(d) $8 \%$ shear strain

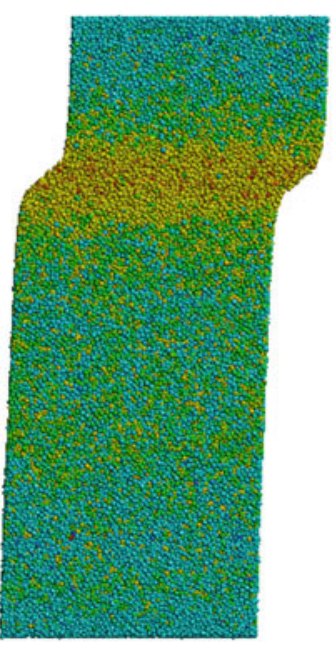

(f) $12 \%$ shear strain

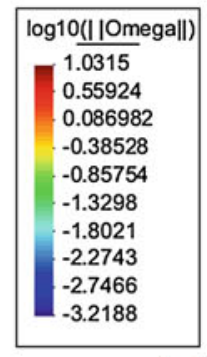

GiD

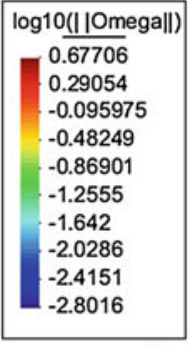

GiD

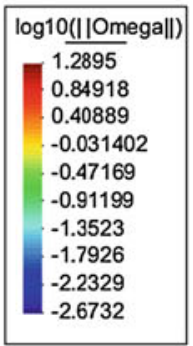

GiD

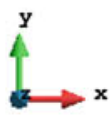

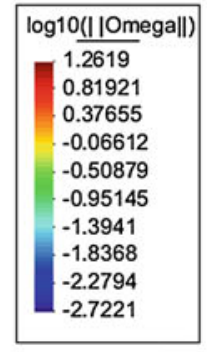

GiD

Fig. 7 Spatial distribution of rotation in assembly at shear strains $=2,4,6,8,10$ and $12 \%$. Color indicates rotation (radians) of individual grains 


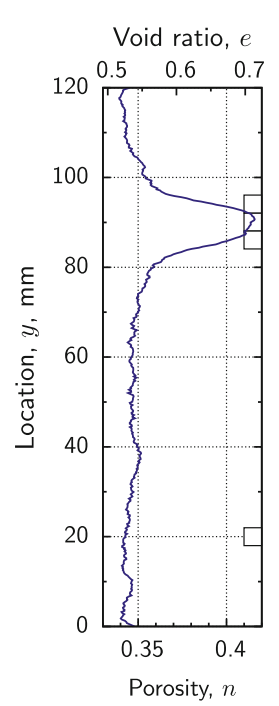

(a)

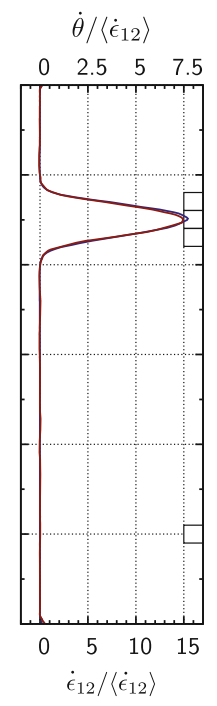

(b)

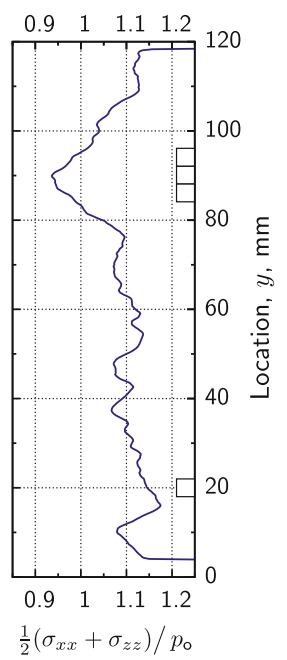

(c)
Fig. 8 Profiles of density, deformation, and confinement along the assembly height a porosity and void ratio, $\mathbf{b}$ shear deformation $\Delta \varepsilon_{x y}$ and particle rotation $\Delta \theta_{z}$, and $\mathbf{c}$ horizontal confinement stress. Boxes along right margins show locations of $4 \mathrm{~mm}$ unit cells for pore analysis (Sect. 3.2)

its pre-peak condition, as that material elastically unloads while softening occurs within the band.

Figure $8 \mathrm{~b}$ shows the profiles of two micro-scale measures of continuum deformation: the local shearing strain $\varepsilon_{x y}$ and the mean rotation $\omega_{z}$ of particles along the height of the assembly. This figure records the differences of strains and rotations between two reference strains, $\varepsilon_{x y}=10$ and $12 \%$. The $2 \%$ increment is the average strain increment $\left\langle\Delta \varepsilon_{x y}\right\rangle$ of the entire assembly, but strains near the center of the shear band are more than 15 times larger than the assembly strain, whereas strains outside of the shear band are nearly zero. The mean particle rotations are also shown in Fig. 8b. The rotations exhibit considerable scatter, as is apparent in Fig.7, and within the band, the standard deviation of these rotations is more than 5 times their mean value. Separate scales are used in Fig. $8 \mathrm{~b}$ for the mean shear strain and particle rotation, since the rotation is half of the strain rate $\dot{\varepsilon}_{x y}$. The lines for shear strain and particle rotation are nearly identical, showing that micro-rotation and vorticity are nearly equal within the shear band.

Figure $8 \mathrm{c}$ gives the profile of the mean horizontal stress, $\left(\sigma_{x x}+\sigma_{z z}\right) / 2$, at the strain $\varepsilon_{x y}=12 \%$, in which the average horizontal confinement stress has been normalized with respect to the initial mean stress $p_{\mathrm{o}}=416 \mathrm{kPa}$. The average horizontal confinement throughout the assembly is $12 \%$ larger than the initial value (also see Fig. 4). The general increase in the average confinement can be interpreted as a response to a dilational tendency that is permitted expression only in the vertical direction, while no horizontal strain is allowed in the $x-x$ and $z-z$ directions. Figure $8 \mathrm{c}$ shows, however, an reduction in the horizontal confinement within the shear band, where the horizontal stress is about $6 \%$ less than the initial stress. This different behavior within the band indicates that mechanical behavior within the band has diverted from the pre-peak response and from the response of material outside of the shear band.

\subsubsection{Fabric of contact orientation}

A common measure of particle arrangement is the distribution of the center-to-center orientations of pairs of contacting particles [31]. During deformation, contacts are more likely to be oriented in the direction of the major principal compressive stress. Figure 9 shows the distributions of the orientations within the $x-y$ plane for the initial, unloaded assembly and at strain $\varepsilon_{x y}=12 \%$, within and outside of the shear band. The initial distribution is isotropic, a result of the isotropic compaction of the specimen. Upon shearing, the distribution becomes highly anisotropic with far more contacts in the direction of the major compressive stress, $\sigma_{1}$, than in the orthogonal direction. Distributions inside and outside of the shear band also differ,

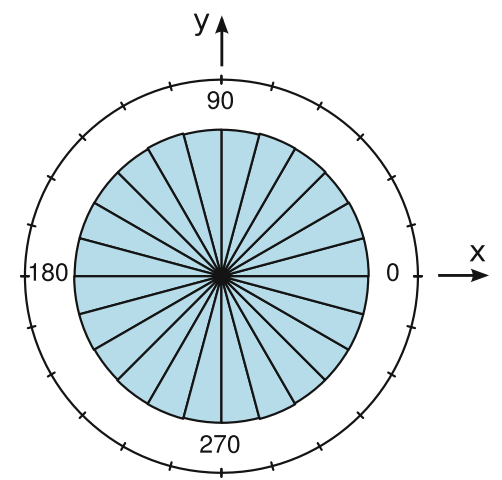

(a) $\varepsilon_{\mathrm{xy}}=0$

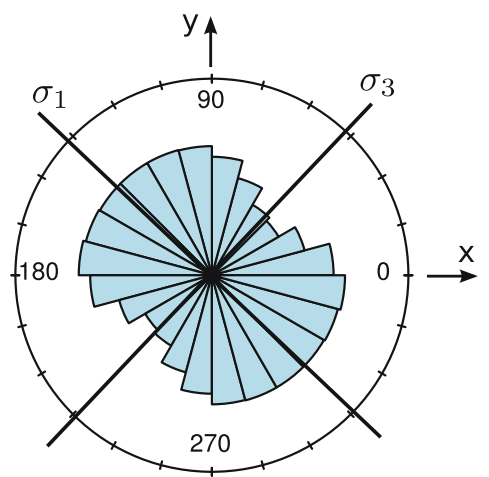

(b) $\varepsilon_{\mathrm{xy}}=12 \%$ within shear band

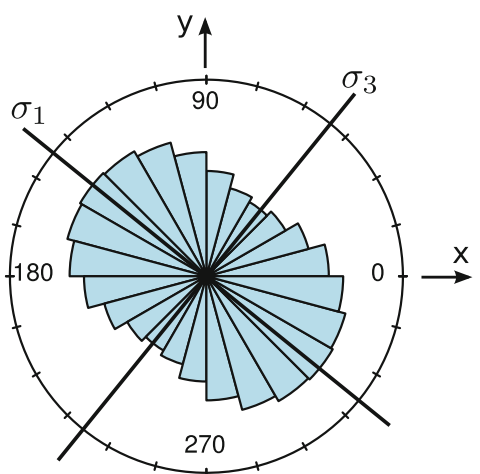

(c) $\varepsilon_{x y}=12 \%$ outside of shear band

Fig. 9 Rose diagrams of contact orientations 
with a slightly greater fabric asymmetry outside the shear band. The figure also shows the directions of the major principal compressive stress, differing by about $4.5^{\circ}$ between material within and outside the band, the latter having a more horizontal orientation. The principal directions of the contact fabric, as defined by [35], are closely aligned with the principal stress directions and differ in a similar manner within and outside the shear band. These results are further evidence that material behavior inside of the shear band has diverted from that of material outside of the shear band.

\subsection{Pore geometry and homogenized hydraulic properties of shear bands}

To analyze effect of strain rotation on permeability, we located the region of grains with the largest rotation (defining the center of the shear band) and nearby, surrounding grains within a $6 \mathrm{~mm}$ distance. The grains with the maximum rotation are at $(20.7,90.1,5.7 \mathrm{~mm})$ relative to the origin shown in Fig. 2. As shown in Figure 8, this location is approximately at the mid-plane of the shear band.

We convert the shear band grain assembly into cubic binary images of size $12 \mathrm{~mm} \times 12 \mathrm{~mm} \times 4 \mathrm{~mm}$. To maintain computational efficiency, we then split the domain into nine $4 \mathrm{~mm} \times 4 \mathrm{~mm} \times 4 \mathrm{~mm}$ unit cells, with each cell containing about 107 particles. For comparison purposes, we also extract the pore geometry of three other adjacent $4 \mathrm{~mm} \times 4 \mathrm{~mm} \times 4 \mathrm{~mm}$ unit cells centered at $(16.7,25.0,5.7 \mathrm{~mm}),(20.7,25.0,5.7 \mathrm{~mm})$, and $(24.7,25.0$, $5.7 \mathrm{~mm}$ ) and labeled them as "host matrix" (see Figs. 2, 8). Samples of binary images obtained from the DEM grain assemblies are shown in Fig. 10. The resolution of the binary images is $0.05 \mathrm{~mm}$ per voxel.
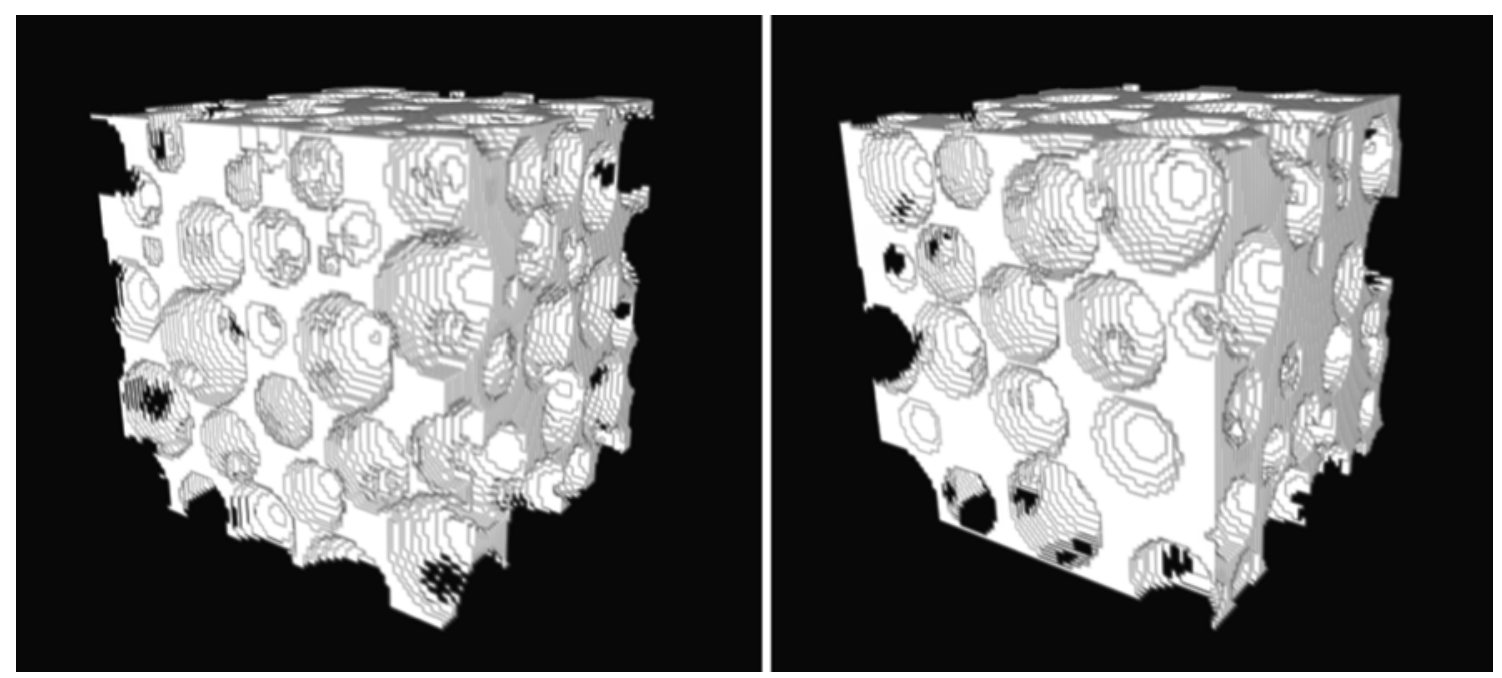

Fig. 10 Binary images generated from DEM assemblies outside (left) and inside (right) shear band at shear strain $\varepsilon_{x y}=12 \%$. Each cell contains about 107 particles 
Table 1 Centroids of the $4 \mathrm{~mm} \times 4 \mathrm{~mm} \times 4 \mathrm{~mm}$ unit cell specimens at which pore-scale flow simulation is conducted to obtain effective permeability

\begin{tabular}{llll}
\hline Location & Coordinate $\mathrm{x}$ & Coordinate $\mathrm{y}$ & Coordinate $\mathrm{z}$ \\
\hline Host matrix & 16.7 & 20 & 5.7 \\
Host matrix & 20.7 & 20 & 5.7 \\
Host matrix & 24.7 & 20 & 5.7 \\
Upper transition zone & 16.7 & 94.1 & 5.7 \\
Upper transition zone & 20.7 & 94.1 & 5.7 \\
Upper transition zone & 24.7 & 94.1 & 5.7 \\
Shear band & 16.7 & 90.1 & 5.7 \\
Shear band & 20.7 & 90.1 & 5.7 \\
Shear band & 24.7 & 90.1 & 5.7 \\
Lower transition zone & 16.7 & 86.1 & 5.7 \\
Lower transition zone & 20.7 & 86.1 & 5.7 \\
Lower transition zone & 24.7 & 86.1 & 5.7 \\
\hline
\end{tabular}

strain. We notice that fluctuation of energy dissipation rate is large when the numerical specimens are less than $1 \mathrm{~mm}^{3}$, but gradually decreases as the sample size get larger. (A $1 \mathrm{~mm}^{3}$ specimen will encompass, on average, only 1.7 particles.) When the size increases to $10 \mathrm{~mm}^{3}$, the energy dissipation per unit volume begins to stabilize. Although energy fluctuation has not yet fully diminished for the unit cell extracted at $12 \%$ shear strain, we decided to use specimens of $64 \mathrm{~mm}^{3}$ to strike a balance between computational efficiency and accuracy.

\subsubsection{Pore geometry characteristics of shear band and host matrix}

Figure 12 summarizes the geometrical measurements and permeabilities calculated via lattice Boltzmann simulations from ten $4 \mathrm{~mm} \times 4 \mathrm{~mm} \times 4 \mathrm{~mm}$ cubic unit cells: three are located at the mid-plane of the shear band (labeled as "shear band"), six are in a transitional zone near the edge of the shear band (labeled as "transition zone"), and three are in the host matrix (labeled "host matrix", see Figs. 2, 8 ). Table 1 summarizes the locations of the unit cells.

Calculations on the binary images reveal that porosities of all unit cells inside the shear band increase from around 0.34 to above 0.4 in the course of shearing from $\varepsilon_{x y}=0$ to $12 \%$ (also see Fig. 8). After the formation of the dominant shear band at strains greater than $8 \%, 5$ out of 6 unit cells stop increasing in porosity. On the other hand, porosity in host matrix increases from initial 0.337 to 0.342 , only a $1.5 \%$ increase during the course of shearing.

The Euler number of the $64 \mathrm{~mm}^{3}$ unit cells, which characterizes the complexity of pore network topology, decreases between the strains $\varepsilon_{x y}=0$ to $4 \%$. This decrease indicates that flow paths become more network-like between these strains. However, as the shear band begins to form and the fraction of sliding contacts drops abruptly at around $5 \%$ strain (Fig. 6b), the Euler number increases steadily from around -500 to -250 . This suggests that the pore-fluid flows through larger pore spaces with less branching of the flow.

The ratio of surface area/pore volume (a measure of pore tortuosity) of the host matrix fluctuates in a narrow range between 11.5 to $12 \mathrm{~mm}^{-1}$ throughout the shearing, whereas, within the shear band, the surface area/pore volume decreases from around 12.5 to $10 \mathrm{~mm}^{-1}$ between the strains $\varepsilon_{x y}=0$ to $8 \%$ and then fluctuates at around 10 $\mathrm{mm}^{-1}$ thereafter. These changes are relatively minor, suggesting that the geometrical tortuosity of the flow channel remains similar throughout the shear test. This trend may be attributed to the uniform spherical shape of the grains and the disallowing of grain crushing in the discrete element simulations.

\subsubsection{Unit cell and multiscale permeability calculations}

The unit cell permeability calculation shown in Fig. 12d reveals that permeability (normal to the shear band, in the $y$ direction) inside the shear band is between 2 and 2.5 times larger than within the host matrix. This result is consistent with the flow streamlines inside and outside the shear band shown in Fig. 13, which indicate that flow velocity within a unit cell inside the shear band is higher than that of the host matrix if given the same prescribed pore pressure gradient.

We also perform regression analysis to check how well the porosity and permeability inferred from binary images correlate with the Kozeny-Carman equation, i.e.,

$k=k_{o} \frac{\phi^{3}}{(1-\phi)^{2}} ; \quad k_{o}=f(s) \mathrm{d}^{2}$

where $d$ is the grain diameter and $f(s)$ is the shape factor, which accounts for the influence of the shape of the pore geometry on permeability. The best fitting curve is shown in Fig. 14. We found that $k_{o}=5,231$ Darcy with $95 \%$ confidence bounds of $[5,122,5,340]$.

Next, we use this $k_{o}$ value to back calculate the inferred mean diameter via a modified Kozeny-Carman equation for which $d=\sqrt{180 k_{o}}$ (cf. [4]). Our intention is to test the importance of changes of pore geometry on the permeability. By treating $k_{o}$ as constant, the inferred mean diameter is $0.964 \mathrm{~mm}$, with $95 \%$ confidence bounds of [0.9539, $0.974 \mathrm{~mm}$ ], a diameter that is only $3.6 \%$ smaller than the median particle size $D_{50}=1.0 \mathrm{~mm}$ and is about $28 \%$ larger than the effective size $D_{50}=0.754 \mathrm{~mm}$. This result suggests that pore shape is not an important factor leading to significant permeability changes inside the shear band. This conclusion is attributed to the fact that the grain assembly is composed of spheres that are rotationally invariant. Whereas sphere rotations may cause relative contact 


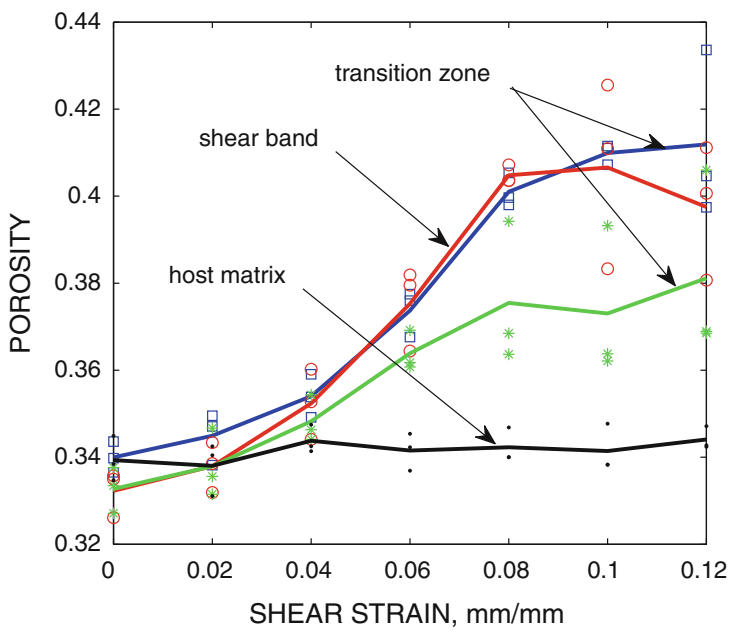

(a) Porosity

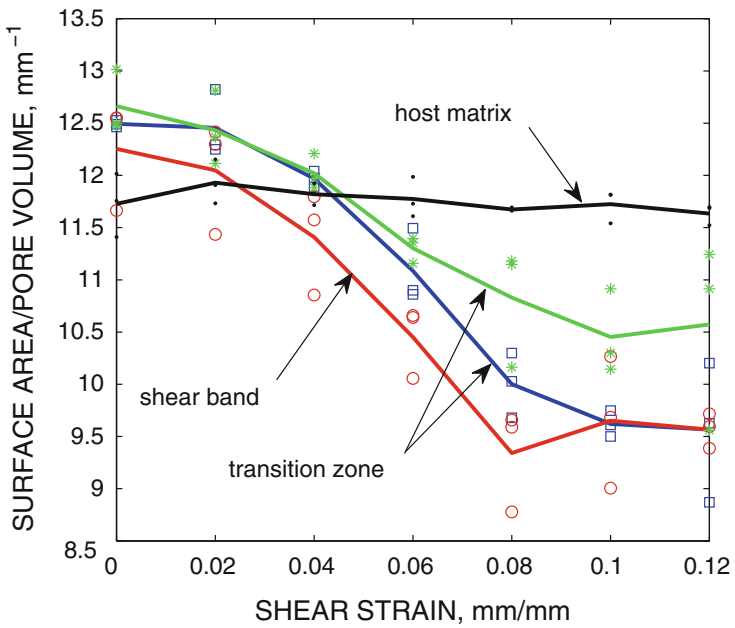

(c) Surface area/pore volume

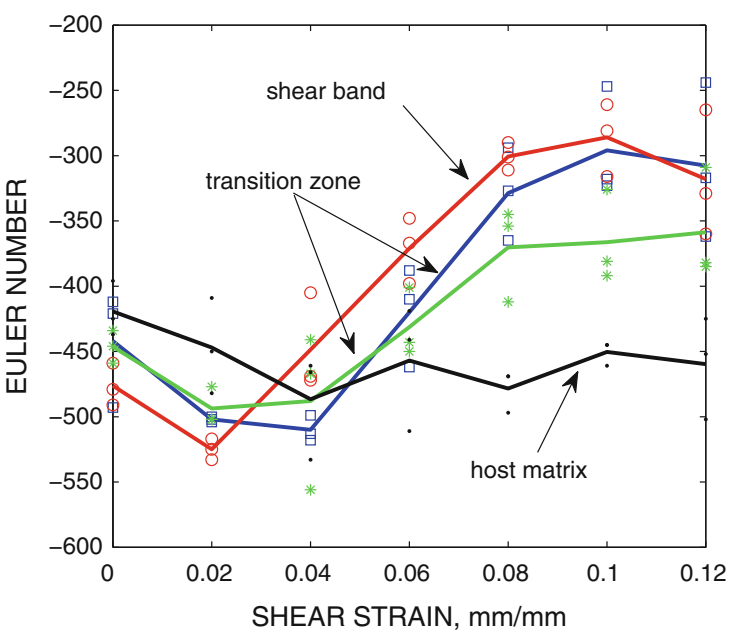

(b) Euler number

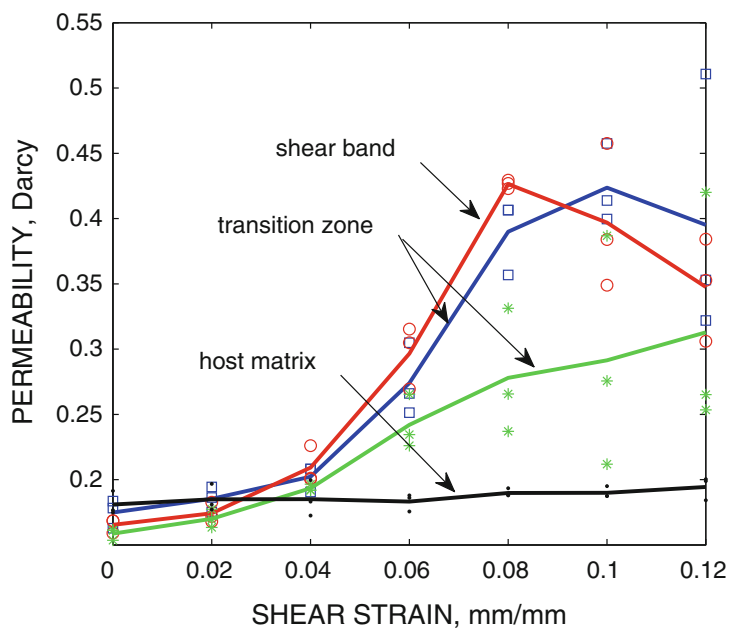

(d) Permeability

Fig. 12 Geometrical measures and estimated permeability computed from binary images generated inside and outside shear bands. Red circles represent data from the middle plane of shear band $(y=90.1 \mathrm{~mm})$; blue squares represent data from the lower transition zone $(y=86.1 \mathrm{~mm})$; green stars represent data from the upper transition $(y=94.1 \mathrm{~mm})$; black dots represent data from the host matrix $(y=20 \mathrm{~mm})$. The lines are the averaged value of the unit cell data with the corresponding colors

displacements and enlarge the pore volume, the rotations of individual spheres do not change the pore geometry. This finding is consistent with the small changes in surface area/ volume and Euler number presented in Fig. 12.

The coefficient of determination $R^{2}$ of Eq. 15 is 0.939 . The relatively narrow confidence intervals and the high coefficient of determination indicate that Kozeny-Carman equation is an appropriate model to correlate permeability with porosity for the DEM assemblies. This result is expected, as the microstructures inside and outside the shear band are both composed of ideal spheres with little overlapping (as indicated by the large porosity). We note, however, that this simple mechanism may not be sufficient to explain the permeability changes if other microstructural attributes such as grain shape, grain size distributions are significantly varied or if when grain crushing occurs.
Assuming that the permeability tensor is isotropic, we conduct a finite element simulation to obtain the effective permeability of the large specimen which consists of the $3 \times 3 \times 1$ contiguous unit cells inside the shear band. For comparison, we also upscale the unit cell permeability by volume averaging and geometric averaging, i.e.,

$\bar{k}=\frac{1}{V} \int_{\mathcal{B}} k \mathrm{~d} V \quad$ (volume averaged permeability)

$\bar{k}=\exp \left(\frac{1}{V} \int_{\mathcal{B}} \log (k) \mathrm{d} V\right)$

(geometric averaged permeability)

These two methods are often used as alternatives to the inverse problem $[4,20]$. Figure 15 compares the effective 


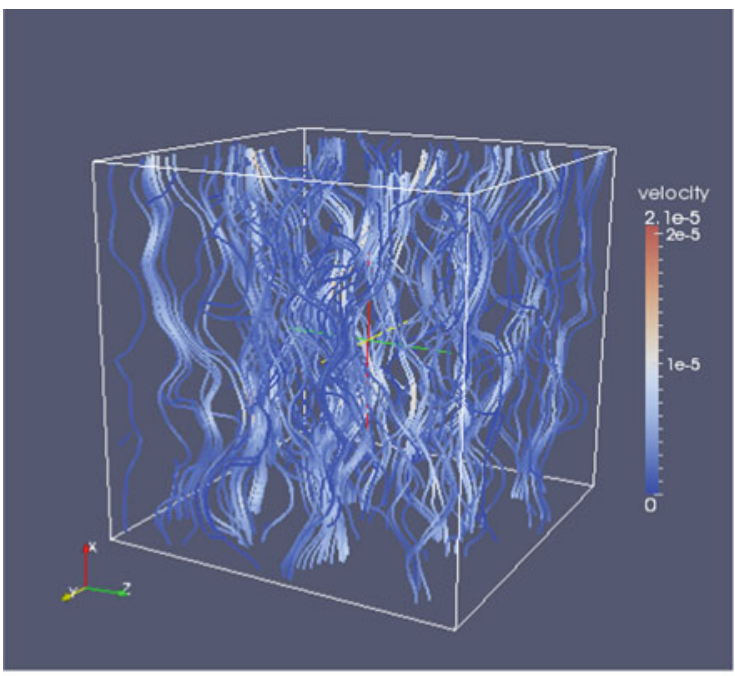

(a) $2 \%$ shear strain (inside Shear Band)

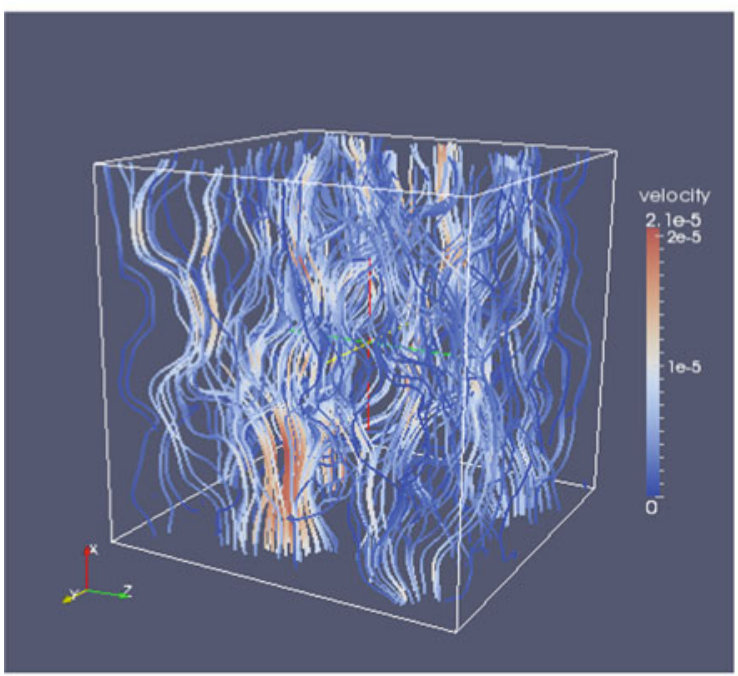

(c) 6\% shear strain (inside Shear Band)

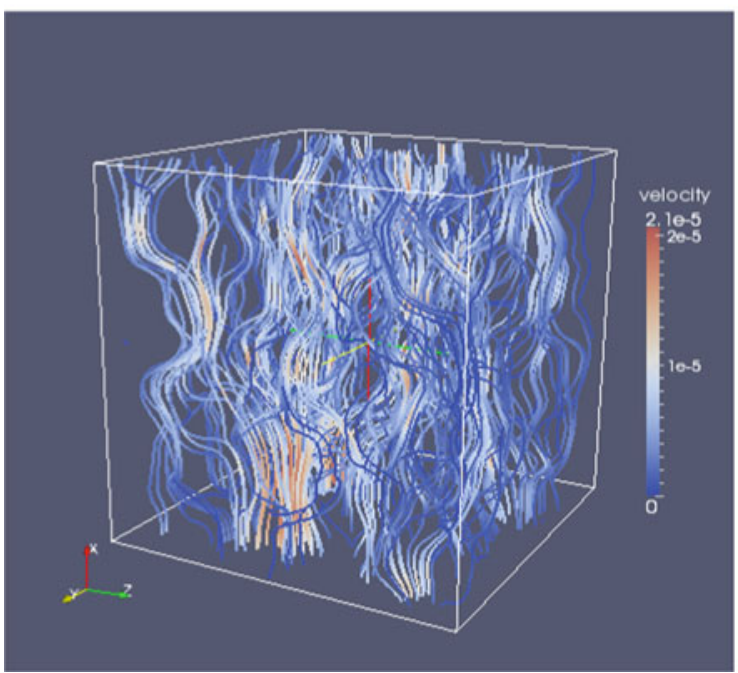

(e) 12\% shear strain (inside Shear Band)

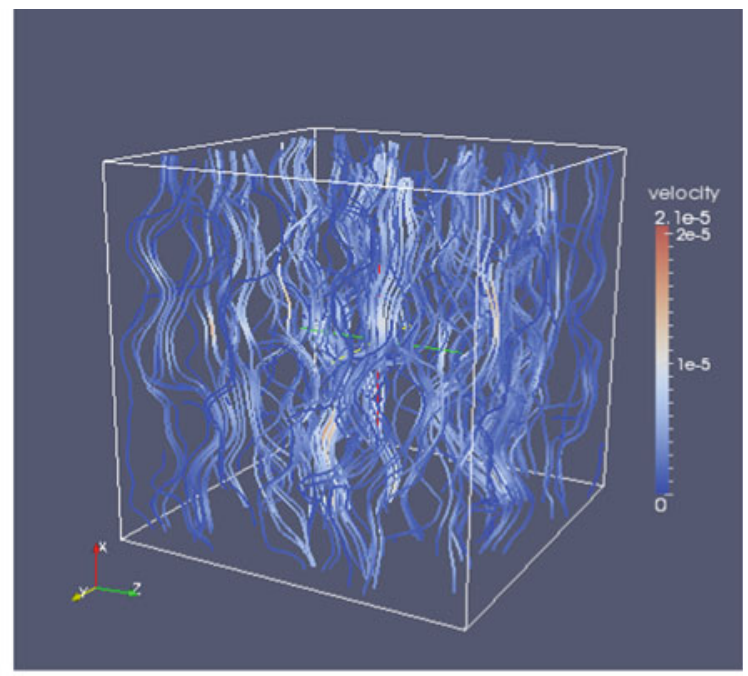

(b) $2 \%$ shear strain (outside Shear Band)

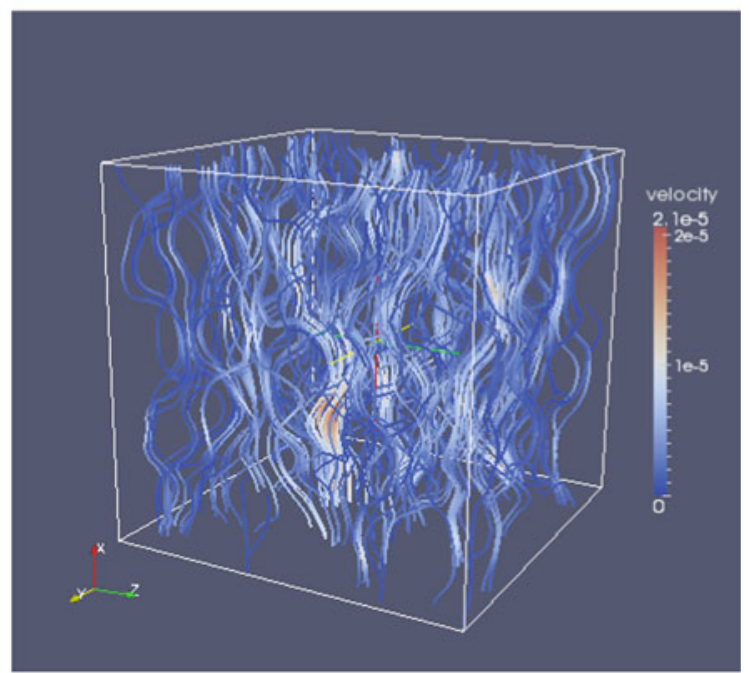

(d) $6 \%$ shear strain (outside Shear Band)

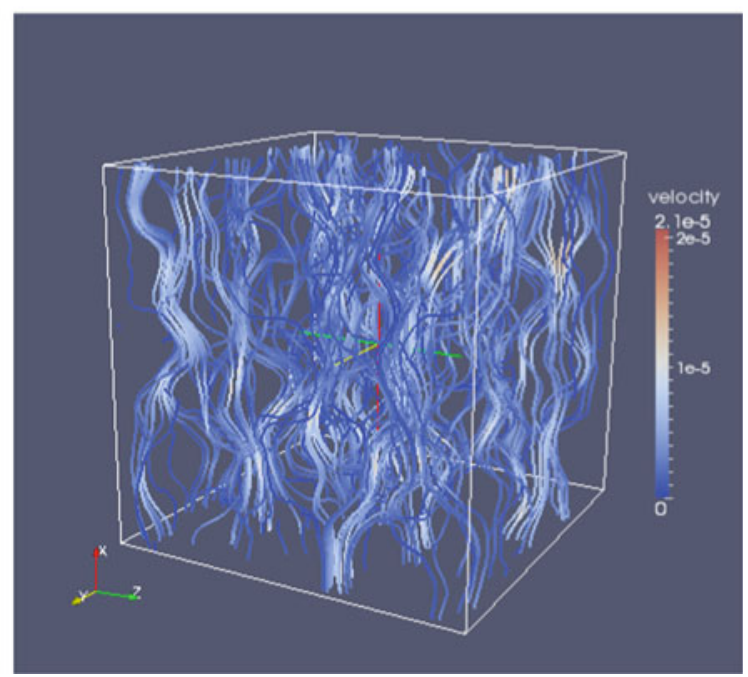

(f) $12 \%$ shear strain (outside Shear Band)

Fig. 13 Streamline of two pore volumes at shear strain $=2,6$, and $12 \%$. Color indicates magnitude of velocity 


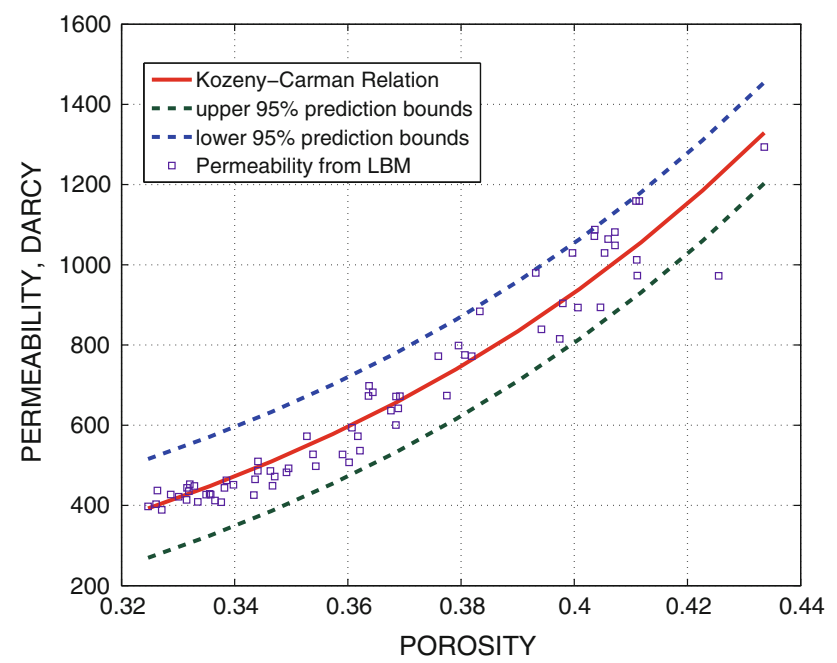

Fig. 14 Regression analysis on Kozeny-Carman relation

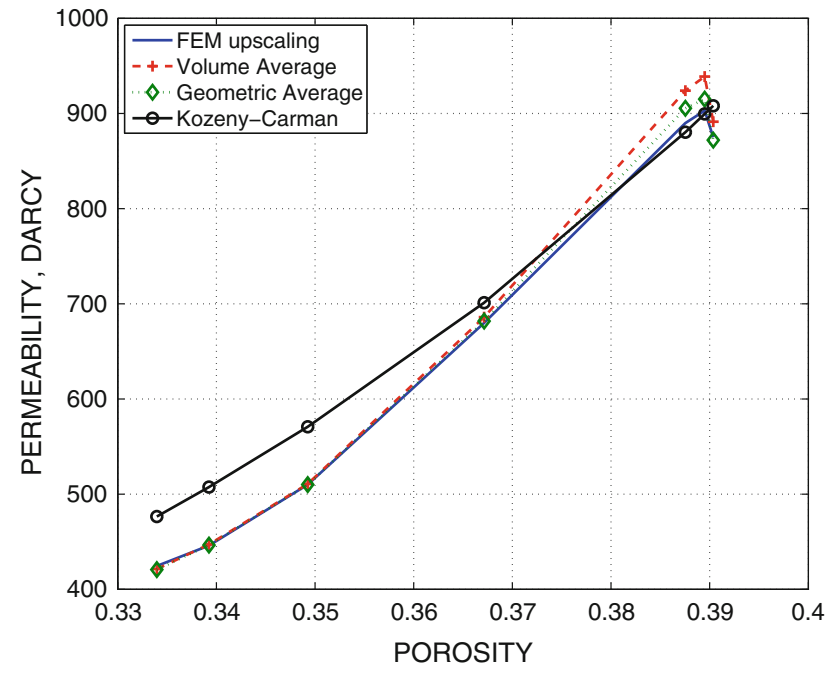

Fig. 15 Effective permeability of the large shear band specimen obtained by FEM upscaling (blue), volume averaging (red), geometrical averaging (green), and Kozeny-Carman relation (black)

permeability calculated by finite element method, volume averaging, geometrical averaging, and the Kozeny-Carman relation. We found that effective permeabilities obtained by geometric averaging are the closest to those inferred from finite element solutions. On the other hand, volume averaging seems to overestimate the effective permeability at higher porosity. This overestimation may be due to the fact that the permeability field is much more heterogeneous at higher porosity, as shown in Fig. 12.

\subsection{Anisotropy of pore space in DEM assemblies}

In order to quantify the anisotropy of the permeability, we compute permeabilities parallel to the shear band (in the

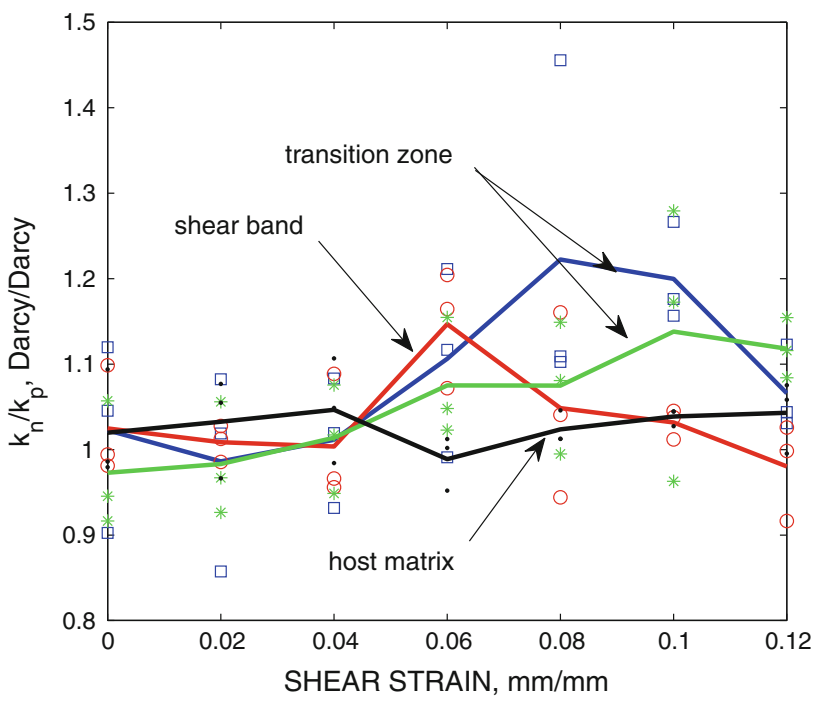

Fig. 16 Ratio between permeability in directions normal and parallel to the shear band. Red circles represent permeability ratio of the middle plane of shear band $(y=90.1 \mathrm{~mm})$; blue squares represent permeability ratio of the lower transition zone $(y=86.1 \mathrm{~mm})$; green stars represent permeability ratio of the upper transition $(y=$ $94.1 \mathrm{~mm})$; black dots represent permeability ratio of the host matrix $(y=20 \mathrm{~mm})$. The lines are the averaged value of the unit cell data with the corresponding colors

$x$-direction) and compare them with permeabilities in the normal direction (the $y$-direction).

Figure 16 shows the ratio between permeability normal and parallel to the shear band. Although the permeability inside the shear band tends to exhibit slightly larger anisotropy, the anisotropies of both the host matrix and shear band are both less than $20 \%$. This result suggests that pore space of the spherical grain assembly remains nearly isotropic even after undergoing significant shear.

\section{Concluding remarks and future perspective}

We present a multiscale lattice Boltzmann/finite element numerical method that quantitatively links particulate mechanics to macroscopic mechanical and hydraulic properties of a grain assembly obtained from discrete element simulations. Using a seed-filling algorithm, we provide a new method to convert DEM assemblies into binary images. This treatment enables us to analyze hydraulic and geometrical attributes of pore geometry in DEM assemblies with unprecedented detail. To the best of our knowledge, this work is the first example of applications of lattice Boltzmann simulations and geometrical measurement on binary images of DEM assembly.

The DEM simulations demonstrate the manner in which deformation and micro-rotation are localized within a shear band. A persistent shear band forms when shearing stress is 
near its peak. Subsequent dilation occurs predominantly within the shear band, leading to larger porosities than within the surrounding matrix. Contact sliding is also concentrated within the shear band. Vorticity nearly ceases outside of the shear band, and within the band, vorticity and mean grain rotation are nearly equal. The DEM results suggest that mechanical behavior within the band diverges from its pre-peak behavior and from the post-peak behavior outside of the band: the mean confinement stress diminishes within the band, whereas it increases elsewhere. Principal directions of contact fabric and stress coincide within the band, although the directions differ significantly from those outside the band. Although loading does induce considerable anisotropy in the contact fabric-the orientations of contacting pairs of particles-the resulting anisotropy of permeability is, at most, modest in these simulations of spherical particles.

We found that dilatancy inside shear band not only increases porosity, but leads to a more interconnected pore space (as indicated by the increased Euler number) and less torturous flow paths (as indicated by the decrease in surface/volume number). These geometrical changes in pore pace induced by shear band formation lead, in turn, to an increase in effective permeability.

Under the idealized situation in which assembly is composed of spherical grains, we find that porosity increase is the dominant factor causing the permeability change. Although geometrical measures do confirm changes in pore geometry within a shear band, the influence of these changes on permeability is minor.

Notice that the proposed numerical framework can also be integrated with laboratory and field measurements. For example, one could imagine calibrating the DEM models with the pre- and post-loading scans and using the numerical method to infer the evolutions of force chain and permeability in between. However, since real particles are rarely in perfectly spherical shape, a more sophisticated method, such as the potential particle approach in [18], must be used to faithfully replicate the geometrical attributes of individual grains in the assemblies. This integration between experimental measurements and numerics is outside the scope of this work, but will be considered in future studies.

Since it is likely that shape changes on pore geometry may play a bigger role if the assembles are composed of nonspherical grains, we are currently investigating the effects of particle shape on hydraulic properties by extending the study to DEM assemblies composed of ellipsoids.

Acknowledgments The authors gratefully acknowledge the support provided by the Geosciences Research Program of the U. S. Department of Energy under Grant No. DE-FG02-08ER15980 to Northwestern University. We also thank Professor Teng-fong Wong for fruitful discussion. We thank Professor Ronaldo I Borja and the anonymous reviewer for helpful suggestions that improved the paper.

Sandia National Laboratories is a multiprogram laboratory managed and operated by Sandia Corporation, a wholly owned subsidiary of Lockheed Martin Corporation, for the U.S. Department of Energy's National Nuclear Security Administration under contract DE-AC0494AL85000.

\section{References}

1. Andò E, Hall SA, Viggiani G, Desrues J, Bésuelle P (2012) Grain-scale experimental investigation of localised deformation in sand: a discrete particle tracking approach. Acta Geotech $7: 1-13$

2. Antonellini M, Pollard DD (1995) Distinct element modeling of deformation bands in sandstone. J Struct Geol 17:1165-1182

3. Aydin A, Borja RI, Eichhubl P (2005) Geological and mathematical framework for failure modes in granular rock. J Struct Geol 29:1831-1842

4. Bear J (1972) Dynamics of fluids in porous media. Elsevier Publishing Company, New York

5. Bésuelle P, Rudnicki JW (2004) Localization: shear bands and compaction bands. International Geophysics Series, vol 89, pp 219-321

6. Bésuelle P, Desrues J, Raynaud S (2000) Experimental characterisation of the localisation phenomenon inside a vosges sandstone in a triaxial cell. Int J Rock Mech Min Sci 37(8):1223-1237

7. Borja RI, Song X, Rechenmacher AL, Abedi S, Wu W (2013) Shear band in sand with spatially varying density. J Mech Phys Solids 61(1):219-234

8. Boutt DF, Cook BK, Williams JR (2011) A coupled fluid-solid model for problems in geomechanics: application to sand production. Int J Num Anal Methods Geomech 35(9):997-1018

9. Casagrande A (1936) Characteristics of cohensionless soils affecting the stability of slops and earth fills. J Boston Soc Civil Eng 23:13-32

10. Chen C, Packman AI, Gaillard JF (2008) Using X-ray microtomography and pore-scale modeling to quantify sediment mixing and fluid flow in a developing streambed. Geophys Res Lett $35(14)$

11. Chupin O, Rechenmacher AL, Abedi S (2012) Finite strain analysis of nonuniform deformation inside shear bands in sands. Int J Num Anal Methods Geomech 36(14):1651-1666

12. Cundall PA (1988) Computer simulations of dense sphere assemblies. In: M Satake, JT Jenkins (eds) Micromechanics of granular materials, Elsevier Science Pub. B.V., Amsterdam, pp 113-123

13. Cundall PA, Strack ODL (1979) A discrete numerical model for granular assemblies. Géotechnique 29:47-65

14. El Shamy U, Zeghal M (2005) Coupled continuum-discrete model for saturated granular soils. J Eng Mech 131(4):413-426

15. Feng YT, Han K, Owen DRJ (2007) Coupled lattice Boltzmann method and discrete element modelling of particle transport in turbulent fluid flows: computational issues. Int $\mathrm{J}$ Num Anal Methods Geomech 72(9):1111-1134

16. Hall S, Bornert M, Desrues J, Pannier Y, Lenoir N, Viggiani G, Bésuelle P (2010) Discrete and continuum analysis of localized deformation in sand using $x$-ray ct and volumetric digital image correlation. Géotechnique 60:315-322

17. Hilfer R, Manwart C (2001) Permeability and conductivity for reconstruction models of porous media. Phys Rev E 64

18. Houlsby GT (2009) Potential particles: a method for modelling non-circular particles in DEM. Comput Geotech 36(6):953-959 
19. Irmay S (1954) On the hydraulic conductivity of unsaturated soils. Trans Am Geophys Union 35:463-468

20. Jensen JL (1991) Use of geometric average for effective permeability estimation. Math Geol 23(6):833-840

21. Johnson KL (1985) Contact mechanics, Cambridge University Press, Cambridge

22. Johnson DL, Koplik J, Schwartz LM (1986) New pore-size parameter characterizing transport in porous media. Phys Rev Lett 57:2564-2567

23. Kuhn MR (2005) Scaling in granular materials. In: García-Rojo R, Herrmann HJ, McNamara S (eds) Powders and grains 2005. A.A. Balkema, Leiden, pp 115-122

24. Kuhn MR (2011) Implementation of the Jäger contact model for discrete element simulations. Chem Eng. 88(1):66-82

25. Kuhn MR, Bagi K (2004) Contact rolling and deformation in granular media. Int J Solids Struct 41:5793-5820

26. Legland D, Kiêu K, Devaux M-F (2011) Computation of Minkowski measures on $2 \mathrm{~d}$ and $3 \mathrm{~d}$ binary images. Image Anal Stereol 26(2):1854-5165

27. Lenoir N, Andrade JE, Sun WC, Rudnicki JW (2010) Permeability measurements in sandstones using $\mathrm{x}$-ray ct and lattice Boltzmann calculations inside and outside of compaction bands. Adv Comput Tomogr Geomater. GEOX2010, ISTE \& Wiley, pp 279-286

28. Lindquist WB, Vankatarangan A, Dunsmuir J, Wong T-F (2000) Pore and throat size distributions measured from synchrotron $\mathrm{x}$-ray tomographic images of fontainebleau sandstone. J Geophys Res 105:21509-21527

29. Louis L, Baud P, Wong T-F (2007) Characterization of porespace heterogeneity in sandstone by $\mathrm{x}$-ray computed tomography. Geol Soc Lond Special Publ 284(1):127-146

30. Mitchell JK, Soga K (2005) Fundamentals of soil behavior, 3rd edn. Wiley, New Jersey

31. Oda M, Nemat-Nasser S, Mehrabadi MM (1982) A statistical study of fabric in a random assembly of spherical granules. Int $\mathbf{J}$ Num Anal Methods Geomech 6:77-94

32. Rechenmacher AL, Abedi S, Chupin O, Orlando AD (2011) Characterization of mesoscale instabilities in localized granular shear using digital image correlation. Acta Geotech 6:205-217
33. Rudnicki JW (2004) Shear and compaction band formation on an elliptic yield cap. J Geophys Res 109:B3

34. Rudnicki JW, Rice JR (1975) Conditions for the localization of deformation in pressure-sensitive dilatant materials. J Mech Phys Solids 23:371-394

35. Satake M (1982) Fabric tensor in granular materials. In Vermeer PA, and Luger HJ (eds) Proceedings of IUTAM symposium on deformation and failure of granular materials. A.A. Balkema, Rotterdam, pp 63-68

36. Scheidegger AE (1960) The physics of flow through porous media. University of Toronto Press, Toronto

37. Schneider CA, Rashand WS, Eliceiri KW (2012) Nih image to imagej: 25 years of image analysis. Nat Methods 9:671-675

38. Succi S (2001) The lattice Boltzmann equation. Oxford University Press, Oxford

39. Sun WC, Andrade JE, Rudnicki JW (2011a) Multiscale method for characterization of porous microstructures and their impact on macroscopic effective permeability. Int $\mathrm{J}$ Num Methods Eng 88:1260-1279

40. Sun WC, Andrade JE, Rudnicki JW, Eichhubl E (2011b) Connecting microstructural attributes and permeability from $3 \mathrm{~d}$ tomographic images of in situ shear-enhanced compaction bands using multiscale computations. Geophys Res Lett 38:L10302

41. Talon L, Bauer D, Gland N, Youssef S, Auradou H, Ginzburg I (2012) Assessment of the two relaxation time lattice-Boltzmann scheme to simulate stokes flow in porous media. Water Resourc Res 48:W04526

42. Thornton C (2000) Numerical simulations of deviatoric shear deformation of granular media. Géotechnique 50(1):43-53

43. Thornton C, Randall CW (1988) Applications of theoretical contact mechanics to solid particle system simulation. In Satake M, Jenkins JT (eds) Micromechanics of granular materials, Elsevier Science Pub. B.V., Amsterdam, pp 133-142

44. Wawersik WR et al (2008) Terrestrial sequestration of $\mathrm{CO}_{2}$ : an assessment of research needs. Adv Geophys 43:97-117

45. White JA, Borja RI, Fredrich JT (2006) Calculating the effective permeability of sandstone with multiscale lattice Boltzmann/ finite element simulations. Acta Geotech 1:195-209 